\title{
Currículo Transversal en la Contemporaneidad
}

\section{Cross curriculum in the contemporary world}

\begin{abstract}
Manuel Antonio Pérez Herrera
Ph.D en Ciencias de la Educación, Rudecolombia. Universidad De Caldas, Manizales, Magister en Educacion y Cognicion. Universidad del Norte ,Colombia Docente Investigador Tiempo Completo,Universidad del Atlántico manuelperez@mail. uniatlantico.edu.co
\end{abstract}

Para citar este artículo: Perez, M. (2016). Currículo Transversal en la Contemporaneidad, Escenarios, 14 (1), p,p 85-101

DOI: http:/ / dx.doi.org/10.15665/esc.v14i1.881

Recibido: febrero 3 de 2016

Aceptado: marzo 18 de 2016

\section{RESUMEN}

El currículum transversal que se aborda en este estudio investigativo apunta de principio a fin a dar cuenta de la orientación social - humana, científica, tecnológica en los estudiantes, la propuesta epistemológica crítica decolonial concibe la comprensión y desarrollo de valores, actitudes, interés y acciones de manera individual y colectiva, posibilitando así formar a los sujetos del saber de manera creativa, camino a la generación de pensamiento crítico, liderazgo social, académico y humano, desde un sistema educativo expedito para el desarrollo integral de proyecto de vida, para lo cual es indispensable la implementación de una metodológica integradora de prácticas, saberes en diversos contextos $\mathrm{Y}$ desde una perspectiva histórica, De este modo, el curriculum transversal, se ubica en el centro del contexto como tal, y desde allí, se abren las rutas de aprendizaje de manera coherente y con identidad en tanto en los contenidos, estrategias, objetivos y demás mediaciones necesarias para hacer posible una formación académica integral de alta calidad, inspirada en la persona como centro del proceso y procurando hacer impacto en el contexto social, teniendo como referencia el contexto social, la investigación, la docencia y la proyección social.

Palabras clave: Curriculum Transversal, Interdisciplinariedad, Transdisciplinariedad, Multidisciplinariedad, Disciplinariedad. Integración curricular, crítica decolonial.

\begin{abstract}
The aim of this research was to know how the transversal curriculum carries out the social, human, scientific, and technological orientation of the students. The proposal critical-decolonial conceives the comprehension and development of values, attitudes, interest, and actions both individually and collectively, so that pupils can be prepared creatively, through the generation of critical thinking; social, academic, and human leadership, in an educational system for their integral development. To get this, it is necessary to implement an integrator methodology of practices, and knowledge in diverse contexts. From that point on, routes of learning are opened coherently and with identity, meanwhile through the contents, strategies, objectives and other means, it is possible to prepare students of high levels, being at the core of that process and trying them to impact the social context. The reference for this will be the context, the investigation, the teaching, and the social projection.
\end{abstract}

Keywords: Transversal Curriculum, Interdisciplinarity, Transdisciplinarity, Multidisciplinarity, Disciplinarity, curricular Integration, critical-decolonial 


\section{INTRODUCCIÓN}

El contexto académico investigativo que fundamentados como: Curriculum Transversal en La Contemporaneidad, su estudio parte de un amplio recorrido crítico, reflexivo, procedimental, conceptual y actitudinal, realizado en posturas epistemológicas, enfoques, marcos teóricos y diseños curriculares caracterizados por la presencia de estructura "disciplinares" - "multidisciplinares" - "interdisciplinares" "transdisciplinar" y "transversales". En tal sentido, desde esos marcos de complejidad curricular, se generaron ambientes académicos provistos de una comunidad educativa coherente que el rol que juega el curriculum en la educación de hoy. El estudio investigativo logra abrir nuevos espacios de encuentro y desencuentro pedagógicos provistos de fundamentos científico - social, concebidos desde prácticas y saberes educativos en contextos reales. Propósito que de principio a fin busco recabar información fidedigna de los análisis de contenidos: (revisión bibliográficas), la observación dirigida y participante articulada en las (prácticas y saberes), por otro lado, el trabajo de sistematización de contenidos y la interpretación comprensiva y propositiva de lo investigado, supuesto metodológico que se concretiza a través de la implementación de una didáctica constructivista alternativa del curriculum, que aunque compleja permitió identificar diversos dilemas, incertidumbres, posturas y actividades educacionales que yacen en curriculum transversal.

Un currículo integral anima a los educadores a contextualizar contenidos, prácticas y saberes sociales y educativos en situaciones reales y concretas y a tomar decisiones a partir de la realidad. El curriculum transversal apunta en primer lugar a atender la diversidad social y la generación de diferentes tipos de desarrollo. Partiendo del hecho de que estos dos ámbitos, son las posibilidades a las que todos los seres humanos tienen derecho, desde la educación media como fórmula de acción social para alcanzar mejor calidad de vida en los ciudadanos. Es en este contexto donde la transversalidad debe ocupar un lugar preferente (Pérez Herrera, 2014). Desde ésta concepción de transversalidad, se conside- ra que el sistema educativo no debe servir solamente para preparar y capacitar a los alumnos en el dominio de ciertas habilidades cognitivas y técnicas instrumentales, sino también a ciudadanos críticos y comprometidos con su realidad social y cultural, es decir, como miembros activos y responsables de su sociedad, su cultura y su mundo (Pérez y Otros, 1994).

\section{Enfoque Metodológico}

En la elaboración, diseño y construcción del curriculum, desde una perspectiva histórica, representar y prever la acción educativa, es una actividad relativamente reciente en el ámbito educativo. Se inicia en la década de los cuarenta y cincuenta en los EEUU, si bien, en (1918) Bobbit William, en su libro The Curriculum estableció las bases para la planificación de la organización escolar, su discurso fue retomado por otros autores. La idea de planificar la enseñanza surgió con la pretensión de hacerla tan eficiente como la producción empresarial, por ello, no es de extrañar que los primeros modelos curriculares generados desde tales presupuestos, imitaron a los modelos empresariales; pero esta no es irremediablemente la única forma de entender el diseño curricular, ya que prever la acción puede entenderse desde otros puntos de vista y desde otra filosofía. Diseñar, planificar son acciones que deben llenarse de contenidos, teniendo en cuenta que existen distintas formas de concebir esos contenidos (Celma, M, 2010: 269).

Es por eso que el sistema metodológico que se implementa en este diseño curricular es pertinente preguntar ¿qué significa diseñar o planear el curriculum? El diseño curricular, constituye un tipo de actividad que consiste no sólo en tomar decisiones que tienen que ver con lo que se quiere conseguir y lo que se hace para conseguirlo, sino que supone también reflexionar sobre por qué se toman determinadas decisiones y se realizan tales o cuales prácticas." Además de las diferentes maneras de entender el diseño curricular y de la complejidad que conlleva, el curriculum se planifica en distintos niveles o ámbitos, que requieren planteamientos y decisiones específicas:

\section{Diversos ámbitos de actuación:}


Ámbito político - administrativo Allí se toma decisiones sobre aspectos generales que afectan al sistema educativo como, por ejemplo, la selección cultural para cada una de las etapas del sistema educativo. En el ámbito del centro educativo como marco general de la vida escolar, se deciden cuestiones que afectan a diferentes cursos y materias de manera global. En el ámbito del aula como espacio de la práctica, de la interacción educativa, se reflexiona sobre alumnos concretos, sobre experiencias particulares, se programan unidades didácticas, etc. Así pues, diseñar el curriculum no es un asunto tan lineal, técnico y concreto como puede parecer, sino una actividad compleja (Celma, M, 2010: 270-271).

En el tema del diseño curricular, es de amplio conocimiento que su planeación se orienta por unos marcos de racionalidad técnica y racionalidad práctica, ámbitos en los cuales hacen su recorrido estructural el Modelo de Racionalidad Técnico (tecnología educativa), cuya fundamentación epistémica está centrada en la formulación de objetivos, elegir experiencias y medios de aprendizaje y la evaluación de resultados. Según este punto de vista, la racionalidad quedará garantizada por una toma de decisiones ajustadamente ordenada y formal (carácter cientificista). Por su parte, los Modelos de Racionalidad Práctica, y, siguiendo algunas concepciones curriculares, que hacen referencia a que:

"Si la secuencia del currículo se invirtiera, es decir, si los maestros fueran invitados primero a experimentar con aspectos específicos del currículo y luego, sobre la base de tales experiencias, se planeara su estructura, la elaboración del currículo adquiriría una nueva dinámica". Taba, H, (1974:23)

Ruta de transición del diseño y la planeación del curriculum Es en esta ruta, que según Fernández Batanero y José María (1992: 4), la transversalidad puede suponer un elemento significativo e interesante como propuesta por dos razones:

1. Considerar la transversalidad como un concepto innovador, por dotar al curriculum de una considerable riqueza y variedad de contenidos que pueden ser organizados en infinidad de for- mas, de manera que den respuesta a las necesidades de los alumnos.

2. Porque las necesidades emanadas de entre el profesorado son síntomas importantes de la resonancia que su propuesta tiene.

La transversalidad debe incitar a toda la Comunidad Educativa a reflexionar sobre la posición que ésta tiene sobre los valores y actitudes, aquellos que quiere transmitir y los que, por otra parte, propugna consciente e inconscientemente la sociedad (Flórez, 1994).

En consecuencia, con el tema del curriculum trasversal, es indispensable abordar la concepción de "interdisciplinariedad", término que es usado indiscriminadamente por la comunidad académica, distinción que es acuñada usualmente en cualquier reunión de especialistas con el fin de analizar determinado problema (Proyecto Académico Curricular, 1999). No obstante, no existe claridad en cuanto a su significado o alcance, lo que atenta contra la posibilidad misma de lograr algún nivel de interdisciplinariedad en el proceso de construcción de prácticas y saberes (Perera C, F. y Otro 1986: 1).

La interdisciplinariedad no puede ser el resultado de una actividad espontánea, aislada y ocasional, sino una de las bases de una concepción pedagógica centrada en el sujeto, meditada, instrumentada y ejecutada por el colectivo pedagógico. La intervención del colectivo no debe limitarse a la relación entre los conocimientos, sino abarcar toda la labor educativa, basada en la propia actuación profesional, la motivación y el ejemplo de los profesores (Flórez, 1999).

Importancia de un currículo interdisciplinar y/o transdisciplinar en la enseñanza - aprendizaje Dado que la interdisplinariedad no solo se basa en las ciencias, sino que transciende los ámbitos disciplinares de manera integral y la transdicplinariedad logra transversalizar prácticas - saberes en contextos reales, a la vez que impacta las mentalidades de los docentes, prepara escenarios para la superación de los obstáculos cognoscitivos, conceptuales, actitudinales y procedimentales que demanda el acto pedagógico. En la enseñanza de las ciencias, la interdisciplinariedad existe en el plano teórico, aunque 
pudieran encontrarse algunas aplicaciones en la práctica, pero estas serían puntuales (Perera C. F. y Otro 1986: 2,3).

En consonancia con lo que se entiende por interdisplinariedad y transdisciplinariedad, se encuentran posturas epistemológicas que ayudan a una mayor comprensión de estos dos conceptos contemporáneos. En tal sentido, Ortega y Gasset (1937) hace más de sesenta años nos prevenía, en la Rebelión de las Masas, lo que denominó "la barbarie de la especialización", "esa casta de hombres sobremanera extraños que son los especialistas. Son aquellos que saben muy bien su mínimo rincón de universo, pero ignoran de raíz todo el resto". Por su parte, Morín (1994), plantea la situación refiriéndose a "la barbarie al interior de la ciencia". Esa barbarie representada en "el pensamiento disciplinar, la compartimentación en disciplinas".

Lo cierto es que los enfoques unidisciplinarios han mostrado su insuficiencia: "su visión reduccionista transforma lo nuevo y complejo en algo simple y corriente, quitándole su novedad y diferencia y convirtiendo el futuro en pasado" (Martínez Miguélez, 2005:2). Uno de los motivos principales, que ha conllevado al predominio de esa escisión curricular, es la tendencia de muchos estudiosos de la ciencia al asumir la investigación desde una perspectiva eminentemente positivista; legitimar el conocimiento de forma reduccionista y precisa, perdiendo su carácter de relevancia (Habermas, 1948).

La anteriores posturas, enrrutan los puntos de vista a analizar comprensivamente en la educación contemporánea, consiste en encontrar las razones del por qué los contenidos seleccionados para orientar la formación de los profesionales, en el tiempo corresponden a caracteres universales, y estos en el recorrido pedagógico han estado y seguirán presentes en los programas educacionales en los Estados y Regiones, partiendo del hecho de que los contenidos son conocimientos fundantes, estructurantes de una disciplina, es decir, conocimientos generativos de una red amplia de conceptos principios, estrategias y problemas y aquellos que por su potencialidad son transferibles a situaciones nuevas.
La educación utilitarista: En el recorrido de la planeación y diseño curriculares, es de sumo interés abordar algunas propuestas curriculares presentadas por didactas y epistemólogos quienes sientan fundamentos conceptuales, procedimentales y actitudinales en el abordaje del currículo desde diversos espacios geográficos del universo académico, en consecuencia: Hilda Taba, Abraham Magendzo, Lawrence Stenhouse, Gimeno Sacristán, Michael Apple, George Posner, Alberto Martínez Boom, Nelson López Jiménez, entre otros; develan la manera como los currículos escolares transitan por diversos modelos, enfoques y diseños que retoman el pensamiento de una educación utilitarista, llena de ideologías, poder y jerarquizaciones de las naciones que han establecido sistemas educativos aun presentes en los modelos educativos más que todo en regiones Latinoamericanas, que divagan en la educación tradicional con enfoques de pedagogía pragmática, y el eclecticismo experiencial y/o experimental misionados por la escuela católica, escuela romántica, escuela desarrollista, etc., las cuales, de alguna manera han sido influenciados por corrientes como la tecnificación de la educación, en la modelación del pensamiento conductual, la formación ética y religiosa, todas asimiladas en la pedagogía intuitiva, transmicionista y receptoras de saberes, en donde se destacan, Skinner, Watson, Santo Tomás, San Agustín, etc. Una educación que apunta a la fragmentación de los sujetos y el creciente esfuerzo misionario como poderosa herramienta para convertir las poblaciones indígenas a la religión de los conquistadores "educación para la conversión religiosa (Michael Mark, 2002: 30).

Los actores curriculares abordados en el párrafo superior, centran sus propuesta de manera alternativa, acogiendo así la transversalidad del currículo también abarca la inclusión y el multiculturalismo, esto en contraposición con un curriculum fragmentado, las propuestas que abordamos tiene una capacidad distintiva de comprensión de poder que emana del currículo, pero que esto a su vez permite hacer de él un instrumento esencial para hablar, discutir y contrastar nuestras visiones sobre lo que creemos de la realidad educativa, cómo damos cuenta de lo que es el presente, de cómo y qué valor tenía la 
escolaridad en el pasado e imaginarse el futuro, al contenerse en él lo que pretendemos que aprenda el alumnado; en qué deseamos que se convierta y mejore (Gimeno Sacristán, 1998: 20).

Otros aspectos del curriculum transdisciplinar: Se considera que un curriculum transdisciplinar, asume a la cultura general como eje articulador y por ende se manifiesta en defensa del multiculturalismo. Además de la inclusión de temas multiculturales refleja su tratamiento en la sociedad, y siempre está presto a acabar con exposiciones superficiales, más que interpretativa o experiencial de otras culturas. En tal sentido, fija su radio de acción tanto en la enseñanza en el aula como las actuaciones sociales de forma contextualizada. Pero lamentablemente, la visión estrecha de multiculturalidad que se tiene en algunos currículos llamados interdisciplinarios o integrales, lo que hacen es reflejar una obligada postura "cosmética", más que una actitud curiosa de reconocimiento de la complejidad que se basa en las experiencias personales de los alumnos y el contexto.

En la "transdisciplinariedad" la acción formativa posibilita el continuo desarrollo progresivo de la ciencia y el desarrollo social (...) bajo la acción integradora de prácticas y saberes, la acción permite observar cambios progresivos en los educandos, en las dimensiones estructurales, curriculares, personales, tanto en sus características individuales como generales y en sus variaciones y complejidades. Se consolida aquí aún más la critica que se le hace a los sistemas educativos reduccionistas de lo científico y lo social, cuando se orientan unos contenidos curriculares donde rara vez, los maestros se aventuran en significados personales o culturales más profundos. Por ejemplo, el meso - contexto, que refleja los valores de la escuela (alfabetización verbal, temas académicos) resuena con el macro - contexto, el énfasis de la sociedad en la alfabetización y su visión de la cultura local - regional, como el desarrollo de la creatividad en los educandos o estratagema para cambiar de ánimo. En el caso de los maestros generalistas, es decir, un maestro que enseña o transmite conocimiento en contextos meso y macro eran a menudo centrales en determinar sus perspectivas y creencias, sobre todo cuando no tenían contextos alternativos.
Proceso de transición Dentro del mismo, es de gran importancia el abordaje del curriculum personalizado. Y en "la concepción de realización personal, a veces denominada curriculum personalizado o curriculum paidocéntrico, el diseñador del curriculum focaliza su quehacer en el conocimiento integral y en identificar necesidades. En otras palabras, los intereses y valores de los agentes del contexto priman sobre los de las disciplinas de estudio e igualmente, las necesidades sociales, los intereses de padres y docentes al momento de tomar decisiones respecto al curriculum (Magendzo, A, 2003:17-19). Aquí se hace visible la autonomía de profesores refleja las relaciones y los valores en la sociedad. La preocupación de los directores por las puntuaciones de los test en las asignaturas académicas refleja el énfasis de la sociedad en la competitividad industrial, el logro material, el dinero y el éxito como símbolo de valía individual y cultural. Dentro de esos valores generales ampliamente aceptados, hay otros sistemas de valores operando: la iglesia y sus nociones de comunidad y espiritualidad, y el contexto del tiempo de ocio y entretenimiento. Por ejemplo, la música escolar igualmente se nutre de estos diferentes mundos. La "cristiandad" ha sido siempre un sistema educacional con Cristo como maestro divino, ordenando a sus discípulos ir a enseñar a todas las naciones (Cremin, 1970).

Elementos históricos y ámbitos internacionales: La educación en el Reino Unido, En Gran Bretaña, Inglaterra y Gales, los Estados Unidos documentada en $\mathrm{n}$ un breve relato cronológico de los acontecimientos de la educación en el Reino Unido en el periodo (1970). Durante esta década hubo cuatro cuestiones principales: una batalla sobre la importancia relativa de enseñar la lectoescritura en comparación con el fomento de la creatividad; el desarrollo de enfoques experimentales hacia la actividad creadora, como de los estilos étnicos; y propuestas para la integración disciplinar. Luego la década de (1980) fue testigo de una creciente demanda de un currículo oficial, con el intento gubernamental de centralizar el poder. Por un lado, pareció que había posicionamiento de la cultura del contexto en el currículo, pero por otro, la posición de la enseñanza - aprendizaje en las escuelas corrió 
peligro al estar organizada por las administraciones locales, con las que el gobierno central estaba en conflicto.

Por otra parte, en la década de (1990) se estableció el currículo oficial, en donde fundamentalmente la práctica social para todos los alumnos de 5 a 14 años, pese a que hubo continúas amenazas a su legítimo estatus en las escuelas y también al futuro de la enseñanza multicultural. Con el nuevo milenio ha habido inicialmente signo esperanzador de "un largamente renacimiento" con la evidencia de un creciente interés por los alumnos, mayor financiación para gestionar proyectos que logren romper las barreras entre las actividades cultuales dentro y fuera de los centros escolares, y una política conjunta para la educación integradora.

De esta manera, se visibiliza como a fínales del siglo $X X$, la prensa se constituye en una fuente inestimable para la fundamentación de los marcos teóricos de la ciencia y la educación integral, ya que proporciona contenidos, comentarios, crónicas y elementos historiográficos continuados sobre los acontecimientos. En Gran Bretaña, la publicación educacional semanal clave es el Suplemento Educacional del Times. Esta proporcionó una valiosa cobertura de las amenazas a muchas disciplinas en las escuelas en (1998) por la preponderancia de las destrezas lingüísticas y numéricas en el currículo.

Sin embargo, en el titular del Suplemento Educacional del Times del (24 de abril de 1998). Un estudio había descubierto con horror que uno de cada cinco colegios de primarios en Inglaterra y Gales estaba reduciendo la enseñanza de disciplinas como las artes integrales, y que algunos centros la estaban eliminando completamente. No había duda de quién era culpable: era la insistencia del gobierno en la enseñanza de las destrezas lingüísticas y numéricas. El Suplemento Educacional del Times organizó una campaña para salvar las escuelas titulada en el "Milenio", y se concentró en escuelas que proporcionaban ejemplos de buenas prácticas culturales. Aparecieron artículos sobre la transferencia del aprendizaje, que sostenían que la investigación científica demostraba que las artes, la cultura y la recreación juegan un papel crucial en el fun- cionamiento del cerebro, y estas disciplinas integradoras ayudan significativamente en el proceso de aprendizaje.

En un curriculum transversal, las fuentes documentales pueden describir una visión de arriba hacia debajo de la historia de la educación, desde las perspectivas de políticos y funcionarios. En la historia de la educación es necesario distinguir la retórica de la realidad, y para esto, las entrevistas a profesores son una forma de hacerlo, es importante saber cómo reaccionan los profesores de secundaria a las nuevas exigencias del currículo nacional, es decir, cumplir con las áreas optativas y reglamentarias de la carta magisterial. En consecuencia, siempre se van a dar opiniones distintas. Por un lado, el trabajo interdisciplinar en el currículo nacional se sugiere ser visto como un progreso académico, mientras que por el otro lado, se percibe como un cambio enorme. $Y$ otros creen que es un progreso que surge de su práctica previa y no de las necesidades y valores del contexto real.

En conjunto, parecía haber pocas dudas acerca de que los profesores están trabajando a gusto con el currículo nacional. Se podría argumentar que este aparente consenso se explica por la evolución del currículo, pero por otro lado, los profesores podrían haber percibido que su asignatura se ha afianzado con su inclusión y que su obligatoriedad para con niños entre 5 y 14 años será vista como un avance importante, cosa que anteriormente se no se consideraba esencial. Siendo así, se amerita de ese maestro comprometido con el rol de una educación de calidad y que apunta a desarrollar en los educandos la imaginación creadora, la convivencia social, así como el cultivo de valores, derechos - deberes y el amor por la identidad local - regional - nacional, son estas y otras finalidades en los sujetos sociales las que contribuyen en su desarrollo integral desde una educación contextualizada, la cual está abierta al cambio, de manera tal que "todos los ciudadanos tengan la oportunidad de recibir una preparación eficiente, eficaz y placentera (Sarró, 1988).

Las consecuencias que generan las creencias de los maestros sobre la cultura en la escuela destaca el rol de ilustrar las materias académicas y 
la función terapéutica de "calmar la bestia en el niño". Contraria a la anterior posición, en el contexto educacional de los Estados Unidos, se presenta un sistema de educación descentralizada, y por lo tal, las reformas curriculares que allí se dan tienen diferentes dinámicas, en comparación con los sistemas más centralizados. La autonomía permitida a los maestros, especialmente, enseñar aquello que más le preocupa y con lo que más cómodo se sienten. Al mismo tiempo, esa autonomía no implica que no se les exija a que se le coloquen más allá de su "zona de confort".

Situación que se consolidad con las aportaciones que se le hacen a la educación con la introducción del modelo de escuela "nueva", desde donde se comienza a dirimir un sistema educativo metodológico que posibilita hacer triangulación entre supuestos epistémicos de métodos científicos (deductivo e inductivo) que toman de las fuentes teóricas y prácticas sociales, haciendo del conocimiento un marco holístico y cultural de posibilidades de desarrollo y asequible para visionar no sólo el conocimiento desde la cientificidad del pensamiento metafísico, sino desde la lógica psicológica del pensamiento, como la transformación de prácticas y saberos sociales.

Desde el ámbito de la "Escuela Nueva", su objeto central es la educación integral, es decir, un sistema abierto mediador de prácticas y saberes sociales, científicos, tecnológicos y de todo tipo de desarrollo humano, ya que quienes participan reaccionan con entusiasmo moderadamente. Aprender en esta escuela tiene paralelismo con la adquisición de múltiples lenguajes, en las que los primeros estadios son intuitivos, y paso a paso se hacen más secuenciales. A pesar de que la concepción conceptual y el desarrollo de destrezas se adquieren en una progresión lógica, y el dominio de técnicas debería estar siempre en función de la experiencia en sí misma a través de la interpretación comprensiva de la realidad social y científica.

En atención a la "Escuela Nueva", en América latina, la historia social de la educación y el curriculum, se inscriben en el proceso de masificación de la educación, en donde adquiere fuerza la idea de que la educación desempeña un pa- pel importante en el cambio social (Magendzo, A: 2003: 14). Por ello, se es consciente de que la cultura general, se debe sustentar en el ámbito de una didáctica social que tiene sus raíces en los discursos culturales y tradiciones, lo cual marca los sistemas de enseñanza-aprendizaje, impactando así como en los valores de la cultura general, ya que en algunos hallazgos de las asignaturas escolares, se observan considerablemente diferencias de alejamiento integral y demarcando así el estatus absoluto más que relativo de esas disciplinas preferenciales dentro del mundo de las escuelas, lo cual tampoco es sorprendente, visión esta que está bien documentado en la literatura académica sobre las asignaturas escolares generales.

En tal sentido, extendemos la crítica reflexiva hacia el sistema curricular oficial y operacional que orienta tanto a maestros generalistas como a especialistas, quienes tienen rasgos comunes en sus prácticas y abordaje de los contenidos. Se visiona que los núcleos temáticos en las asignaturas preferenciales (al igual que en las artes), se fragmenta la acción progresiva de las asignaturas académicas), donde la elección de núcleos temáticos solo se orienta desde disciplinas clásicas, tales como las ciencias básicas, lectura, escritura y algunos temas de civismo y educación moral y religiosa. La mirada que la escuela tiene de la tradición cultural general, así como las creencias y las visiones personales de los maestros individuales casi no se tienen en cuenta al momento de diseñar y poner en ejecución a los currículos nacionales. Situación está que se hace presente en los currículos de muchas regiones Latinoamericanas. (Freire, 1974).

En consonancia, el rol que se ejerce tanto en el currículo oficial, como el operacional, su mayor característica se presenta donde el sujeto en la sociedad está influenciado por un sistema elitista, de recompensas y estatus que devela "la importancia que la sociedad atribuye a ciertas asignaturas que proporcionan recompensas psicológicas adicionales a los maestros, al incrementar el valor atribuido a su labor docente". "Lo contrario, por supuesto, también es verdad: los maestros de asignaturas menos valoradas, a menudo experimentan menos recompensas sociales y psicológicas (Grossman y Stodolsky, 
1997: 26).

Algo muy significativo en la activación de los contextos sociales, tiene que ver con los modos y tensiones culturales, es decir, el suscitar de emociones moduladas por diversos parámetros, por el ejemplo, la educación debe dar cuenta de cómo se da (el estado original de la acción motora - equilibrado en los seres humanos y lo que le proporciona el ambiente social), estas tensiones generan en el ser humano efectos emotivos y afectivos. El estudio de estos parámetros hace posible la identificación de efectos psicológicos - cognitivos producidos y se develan en reacciones de alegría, tristeza o sosiego. (Cox, 1998)

El currículo transversal, igualmente da cuenta de estudios experimentales de la capacidad de evocación emocional algo que es reciente, ya que las emociones transversalizan a los seres humanos, y estas han permanecido por largo tiempo sin ser exploradas por estudiosos de la cognición, pues éstos eludían abordar los aspectos irracionales del comportamiento humano. ¿Acaso no postulaba Descartes que, para penetrar en los secretos de la razón, era necesario excluir antes la pasión? Este principio ha influido desde hace mucho en la forma de articular los conceptos de emoción y de cognición: se presumía que la cognición se encargaba de los procesos conscientes de alto nivel, mientras que la emoción correspondía a mecanismos incontrolables, regidos por el sistema nervioso central (Revista mente y cerebro, 2005: 5).

Se suponía, además, que la cognición y las emociones eran independientes. Sabemos ahora, merced sobre todo a los trabajos neurobiológicos de Antonio Damasio, de la Universidad de Iowa, que la cognición y la emoción se hallan íntimamente vinculadas, y que, por ejemplo, los aspectos emotivos desempeñan un papel determinante en los comportamientos racionales de toma de decisiones. En consecuencia, las respuestas emocionales son reproductibles de unos momentos a otros en una misma persona y entre individuos. Tales resultados son coherentes con la idea de que las emociones aseguran una función de coherencia social en una cultura dada (Revista mente y cerebro, 2005:6).
La capacidad de reconocimiento de las emociones y la cognición, son atribuibles a una educación que forme para la vida a hombres y mujeres en contextos eficaces y placenteros. Una formación que profundice en la historia de la humanidad y se logre la comprensión de la comunicación humana, dialogante y comprensiva. La anterior nos sirve para explicar la importancia social del conocimiento en contexto, el cual está provisto de cohesión social y de "sincronización" anímica individual y colectiva.

El análisis de contenidos realizado sobre el currículo integral, condujo a encontrar información que contribuye a fundamentar el tema de la transversalidad del curriculum, en tal sentido, en el marco de la reforma educativa en curso en la República de Argentina, normada por la (Ley 24195 del año 1993). A partir de la promulgación de la Ley Federal de Educación, numerosos documentos de apoyo han sido elaborados para facilitar su aplicación, hasta llegar a la (resolución 39/94) del Consejo Federal de Educación (CFE) que aprueba los Contenidos Básicos Comunes (CBC) para la Educación General Básica (EGB). Este establece los lineamientos a los cuales deberán ceñirse las instancias Jurisdiccionales (Cuidad Autónoma de Buenos Aires y todas las provincias) para realizar sus diseños curriculares; y las instancias educativas del Sistema General de Educación del País.

Los Contenidos Básicos Comunes (CBC) para la Educación General Básica (EGB) aparecen organizados en capítulos que abarcan los Contenidos de Aprendizaje (CA) a partir de su pertenecía a determinados Campos Científicos o Culturales. Esos capítulos son: Lenguaje, Matemáticas, Ciencias Naturales, Ciencias Sociales, Tecnología, Educación Artística, Educación física y Formación Ética y Ciudadana. Al reañozar el análisis de la fundamentación presentada para otorgar la denominación del Área de Educación Artística al agrupamiento de (CBC) de Música, Expresión Corporal, Teatro y Plástica, se dice que: "pueden ser sólidamente fundamentados como área en el proceso educativo del niño y de la niña desde múltiples perspectivas: la Psicología, dados los procesos interactivos y afectivos que se movilizan al pintar, actuar, cantar y expresar corporalmente; la Psicomotriz, dado que 
se integran todas la dimensiones, del sujeto en el Acto de expresión; la Sociológica para la interrelación de sujetos en Pos de la realización de una producción común, considerando su significación como rito social; la antropológica, que implica las resonancias de la historia de la cultura del ser humano implícitas en todo hecho artístico; y por ultimo, la cultura en tanto productos de la cultura humana, en particular de los grupos en los cuales está insexto el sujeto en su relación con la historia y con la identidad cultural de su comodidad." (Frega, A. 2003: 2 - 3)

En la investigación, fue necesario comenzar a comprender como se encuadra la estructura de los Contenidos Básicos Científicos (CBC) en la educación de Argentina, quien devela como punto de partida un currículo "donde las relaciones entre contenidos, aparentemente no es agregado (pues no están aislados entre sí en una relación cerrada), sino integrado, (pues los contenidos se presentan en una relación abierta entre sí)" ya que "la noción de integración aquí, se refiere mínimamente a la subordinación de materias previamente aisladas, a una idea que las relaciona, lo cual borra la delimitación entre la mismas" (Bernstein, 1985: 2, . En Frega, A. 2003: 5).

Por otro lado, la integración de disciplinas mediante aéreas del conocimiento es una modalidad bastante divulgada y es también la que utiliza la Ley Española (LOGESE, 1992 - 2012) para presentar los contenidos mínimos. "La vertebración se lleva a cabo agrupando aquellas disciplinas que mantienen similitudes importantes en contenidos, en estructuras conceptuales, procedimientos, metodologías de investigación, entre otras“. Desde el punto de vista histórico, la tendencia a la diferenciación del conocimiento en una multiplicidad de disciplinas autónomas comenzó a principios de siglo XIX, vinculado al proceso de transformación social que se dio en países europeos más desarrollados: "Técnicas y Saberes se fueron diferenciando progresivamente $y$, a su vez, los lenguajes que los caracterizaban se fueron especializando y circunscribiendo a ámbitos específicos. Surgió de este modo el concepto disciplinas con un objeto de estudio, - marcos conceptuales, métodos y procedimientos específicos" (Torres, 1998: 58. En Frega, A. 2003: 4-5).
El curriculum como proceso permanente de investigación, en primera instancia debe complejizar las actuaciones, interés, valores y problemas que presenten los estudiantes en general, para que se sientan protagonistas de un sistema educativo incluyente y de paso acabar con la visión fragmentaria, técnica y unilateral de lo humano, en donde lo persistente es la poderosa influencia etnocéntrica de la exclusión y la delimitación tanto de los contenidos y valores del currículo. No es secreto que el siglo XX se destacó por el progreso tecnológico en todos los campos del conocimiento científico, pero su fraccionamiento y separación han impendido a la humanidad ver lo que está entrelazado, reduciendo, como consecuencia, la visión y la comprensión de lo global. En el campo de la educación, a partir de (1990) y bajo la propuesta (LOGSE), se ha planteado una reforma que atañe a todo el sistema educativo y que se hace referencia a un enfoque global e integrador, que en la profesión de la enseñanza se considera relevante y que los alumnos que se preparan para ser futuros profesionales deben dominar el sentido de la integración en el periodo de formación La Ley Orgánica de Mejora de la Calidad Educativa (LOMCE, 2012).

El análisis interpretativo de esta normatividad, conduce a establecer la identidad que tienen algunas regiones de España, como Granada, Navarra, Madrid y de América latina, Argentina, México, Chile, Brasil, etc., quienes en su campo de acción de la (educación infantil), prevalecen las actividades globalizadas como forma de enseñanza, y se acentúa el carácter global e integrador que debe existir en las diferentes aéreas que se imparten.

Mas adelante, esta misma reforma hace referencia a la complementación y el enriquecimiento mutuo de las diferentes posibilidades expresivas y artísticas expresando textualmente: de ahí que sea posible y aconsejable el tratamiento conjunto de los distintos ámbitos y de sus elementos, tratamiento que precisamente su integración en una sola área educativa puede contribuir a favorecer. Por otro lado, La Ley Orgánica de Mejora de la Calidad Educativa (LOMCE, 2012), hace una declaración general de intenciones a favor de una formación integral y global, incluso poli- 
valente, en la formación inicial del profesorado y uno de los elementos que podría contribuir a ello seria, sin duda, la interdisciplinariedad.

La ley manifiesta, que en la educación primaria la educación artística forma parte de las cinco aéreas obligatorias, en las que subraya el carácter global e integrador. Un poco más adelante, esta normatividad precisa que la educación primaria será impartida por maestros que tendrán competencia en todas las aéreas de este nivel. Detallando cuidadosamente lo antes mencionado, podemos apreciar que la educación artística es un área de este nivel. Todo esto una clara imprecisión, porque en realidad maestros de educación artística no existen. Sin embargo, en la ley, teóricamente sí. Además, el único ámbito de la educación artística que aparece legalmente autorizado para impartirse es la música, lo afirma cuando expresa: la enseñanza de la música, de la educación física, de los idiomas extranjeros o de aquellas enseñanzas que se determinen, serán impartidas por maestros con la especialización correspondiente. En ningún momento menciona los otros dos ámbitos correspondientes en el área de educación artística.

La crítica que se le hace a la reforma educativa (LOMCE), es que no contempla el área de educación artística como tal, sino que particularmente se trabaja según lo indica la Ley, es el lenguaje de la música. Tal situación, conduce a formular las siguientes preguntas: ¿cabria un especialista en educación artística? ¿Quién impartirá legalmente la plástica, la literatura, la dramatización? ¿Si legalmente está constituida el área de educación artística porque no existe el maestro correspondiente? ¿Se podrá dar el enfoque globalizador que propugna la ley si no existe el maestro correspondiente? ¿Tendrán competencia los metros que se forman en la Universidad Pública de Navarra (UPNA) para impartir el área de Educación artística? ¿Por qué no se incluye la dramatización en los planes de estudio de que ofrece la UPNA? (Ortiz, 2004: 5-25).

El rediseño curricular y la instrumentalización Un rediseño curricular "reestructura y modifica sustancialmente los requerimientos profesionales". Según Echeverría (2002: 8), lo que demanda una actualización y redefinición de los elementos que inciden en la práctica y desarrollo profesional de cara al diseño de unos programas formativos pertinentes y actualizados son todos aquellos intereses y valores que dignifican la vida de los ciudadanos y/o escolares. Partiendo de la idea de Imbernón (1999) el desarrollo profesional docente está unido a un concepto de profesionalización dinámico, flexible y sensible a los cambios de la situación laboral y social. En tal sentido, Novoa (1999), recomienda construir un saber pedagógico que no sea puramente instrumental sino que tenga en cuenta el componente personal de cada docente, será imprescindible abordar la práctica profesional no sólo desde la descripción y valoración del puesto de trabajo a través del conjunto de conocimientos y competencias necesarias para desarrollar la profesión, sino partiendo de una visión mucho más global en la que se tenga en cuenta el proceso de socialización vivido desde la infancia hasta el momento actual, haciendo referencia a los condicionantes sociales, personales, académicos y del entorno laboral que ha ido configurando la identidad de este colectivo y que han determinado su desarrollo profesional (Ocaña Almudena, 2006: 15).

Un curriculum integrador, es aquel que permanentemente se somete a diagnóstico de necesidades y valores, permite determinar el distanciamiento entre la cultura de la cotidianidad y la prevalencia de la cultura universal como la soberana de un proceso desigual y desconectado de la realidad social (Bernstein, 1985). “El currículo integral" amerita de un proceso de búsqueda de negociación, de valoración, de crecimiento y de confrontación entre la cultura universal y la cultura de la cotidianidad, y la socialización entre la cultura de dominación y la cultura dominada (López, N, 2002:55).

El curriculum transversal, es aquel en donde sus actores logran transformaciones significativas tanto en sus formas de actuar, pensar y hacer, para eso es importante hacer uso coherente de alternativas de cambios que logren minimizar las actuaciones con intencionalidad excluyente y la desigual del mundo moderno (...), ya que lo que requiere el cuestionamiento de las pretensiones de objetividad y neutralidad de los principales instrumentos de naturalización, y legitimación de este orden social (García C, 1984). 
. Y lograr la consolidación del trabajo transversal bien sea desde el conjunto de saberes que conocemos globalmente como ciencias sociales. Este trabajo de reconstrucción es un esfuerzo (...) que se ha venido produciendo en los últimos años en todas partes del mundo (Lander, 2000: 12). (Freire, Paulo, 1974).

En atención a lo explicitado, el proceso de construcción del conocimiento debe entenderse entonces como una actividad compleja de situaciones reales, de búsqueda de soluciones, de análisis y discusiones, de tomas de decisiones por la comunidad educativa y el colectivo estudiantil, propiciando el desarrollo de habilidades investigativas a la par con el conocimiento personal y de grupo (Vega Miche y Corral Ruso, 2006: 8). Frente a lo expuesto, es necesario identificar, si la educación integrada, cuyos contenidos estén orientados con identidad desde una estructura curricular flexible y transformista.

\section{Resultados Obtenidos en el Procesamiento de la Información}

El proceso de investigación curricular está fun- damentado en el paradigma cualitativo, propio de las ciencias sociales, presenta un enfoque descriptivo e interpretativo, el estudio por su naturaleza corresponde a un estudio de caso simple - alternativo, en consecuencia, fue factible la implementación de un sistema metodológico flexible, en el cual se hizo uso de técnicas e instrumentos de corte cuantitativo y cualitativo, lo cual posibilitó la recolección de la información desde distintas fuentes, tales como: el análisis de contenidos, primera fase del estudio que facilitó recabar información teórica - bibliográfica, y, en la interpretación de la información se hizo uso de la triangulación como instrumento mediador, facilitador del manejo de la información compilada y sometida a procesamiento, a confrontación, como a la transferencia de conocimiento y producción teórica. En consecuencia, el curriculum estudiado se dimensiona como "tentativa para comunicar los principios y rasgos esenciales de un propósito educativo de forma que permanezca abierto a discusión y crítica y pueda ser trasladado efectivamente a la práctica" (Stenhouse, 1984:29).

Figura 1Visión holística del curriculum transversal, un diagrama Inter curricular activo

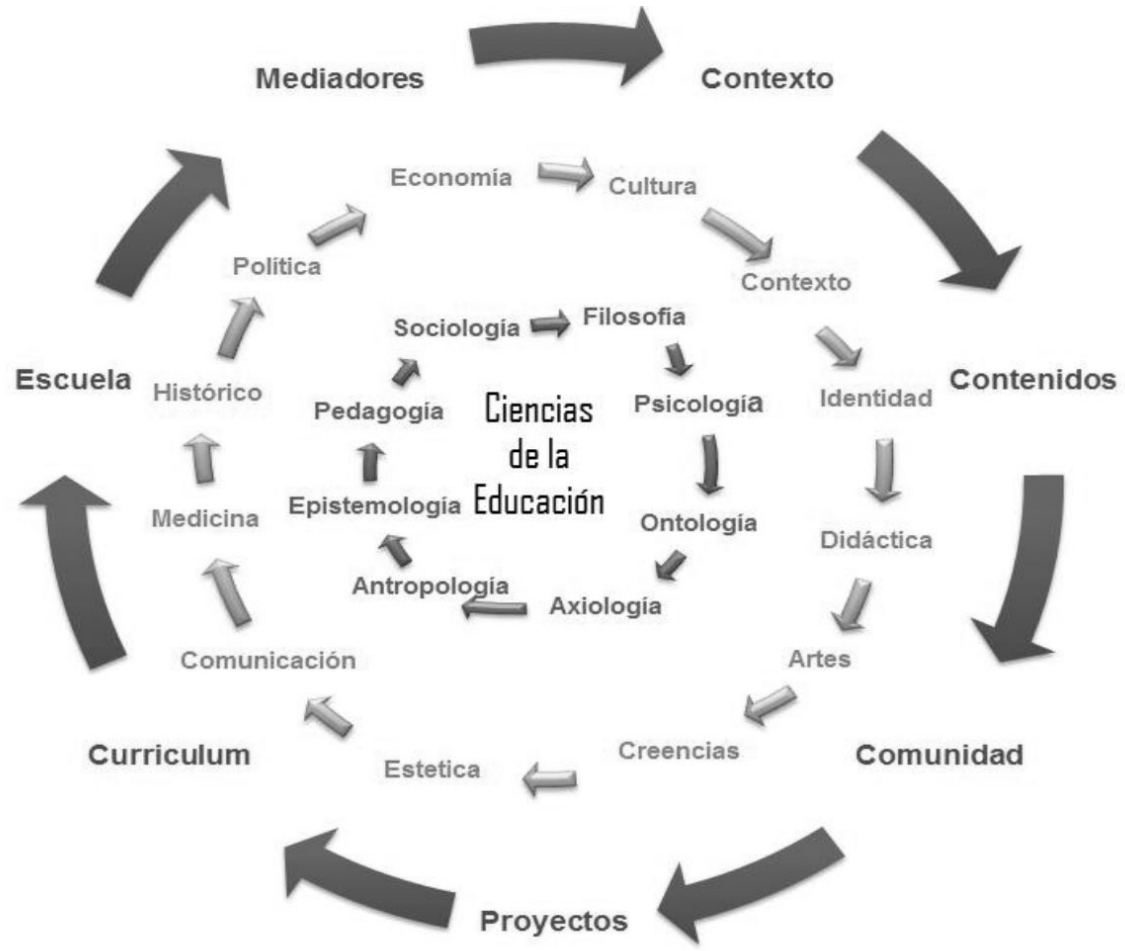

Fuente: Perez Herrera, Manuel, Docente investigador Universidad del Atlántico, Barranquilla, 2014 
ES así que se plantea una visión holística del curriculum transversal, con un diagrama Inter curricular activo Ver fig.1 En el proceso de interpretación realizado en el estudio del curriculum transversal, emergieron las siguientes categorías: Categoría 1: Curriculum Disciplinar. Categoría 2: Curriculum Integral. Categoría 3: Curriculum Interdisciplinar. Categoría 4: Curriculum Transdisciplinar.

Categoría 1: Curriculum Disciplinar: En el recorrido interpretativo comprensivo realizado sobre lo que en el tiempo la comunidad científica ha significado como curriculum transversal y diseño curricular. No obstante, se abrió este debate haciendo una descripción e interpretación sobre las formas tradicionales de organización del conocimiento: la disciplina, como visión unilateral de la realidad, lo cual no agota la comprensión de ella y mucho menos en sus dimensiones complejas. "Adaptar" a la nuevas generaciones a las exigencias de la marcha creciente del desarrollo económico, las aportaciones de la ciencia y la tecnología, la sumisión a una jerarquía, a la separación de la mente y el cuerpo, del trabajo manual y el trabajo intelectual, es obvio que las escuelas han de inspirarse en los principios del paradigma analítico - cartesiano y con ellos conseguir ciudadanos que se ajusten bien a esa engrasada maquinaria que es la economía (Yus Ramos, 1996- 2001:14).

Lo anterior explica la importancia que la escuela le otorga a áreas del saber que son fundamentales para este éxito económico y también explica cómo desde muy temprano se escinden ( y con ello se clasifican al alumnado) las habilidades manuales (destinadas a la clase trabajadora) y las habilidades intelectuales (destinadas a la clase profesional), con tal menosprecio al ámbito de los sentimientos, el de las intuiciones, la fantasía, la espiritualidad, la creatividad, el arte, la música, la expresión corporal y el desarrollo moral, todos estos aspectos fundamentales de la persona quedan en un segundo plano, como máximo opcional o marginal en todos los sistemas educativos, con fuerza creciente conforme crece la edad del estudiante.

Categoría 2: Curriculum Integral. La búsqueda de un planteamiento integrado de la educación, el afán por llegar a esas otras dimensiones, tratando de educar el "todo" de la personalidad, es una tradición que en este ámbito se conoce como Educación Integral, pero que últimamente, y en especial en países anglófonos, se viene conociendo como Educación Holística. Es por ello que, a partir de lo que se supone como un nuevo paradigma que emerge en el siglo XXI, y tras una reflexión y práctica crítica como educadores científicos del sistema educativo materialista y racionalista, últimamente a partir de la tensión generada por esa "otra escuela" que actualmente compite con el academicismo tradicional, representada por los llamados "temas transversales", cuya traducción educativa óptima exigirá un replanteamiento radical de las bases y fundamentos de la escuela actual (Yus Ramos, 1996- 2001:14-15).

En consecuencia, la globalización de la cultura, la educación integral de la persona, la organización democrática de la escuela y el compromiso de la educación con la problemática socio-natural. Estos cuatro ejes que actúan en una matriz moral, que se nutre de una ética de mínimos universalizables y cuya meta podría ser una ciudadanía crítica, radicalmente democrática, que participara en la construcción de un nuevo humanismo ilustrado, que incorporará una tercera generación de derechos humanos. Para ello, es preciso dar un vuelco a la escuela, facilitar al alumnado nuevos marcos de referencia desde los primeros niveles de enseñanza, para permitirle contemplar la dimensión planetaria de la cultura, así como desarrollar un sentido de la responsabilidad y la lealtad con los pueblos y grupos sociales marginales y silenciados. En síntesis, el curriculum integral, esta plantada para una escuela que forme para la vida y donde el estudiantado desarrolle destrezas sociales y políticas, desde una vida escolar profundamente participativa, democrática y comprometida con su medio, para capacitar al alumnado para que participe de forma efectiva y desde una perspectiva autónoma, critica, tolerante y solidaria, en la toma de decisiones ante la compleja globalidad de la problemática socio - natural que afecta hoy y mañana a la humanidad y al planeta.

Categoría 3: Curriculum Interdisciplinar. La 
"interdisciplinariedad", considerada para muchos como la habilidad y práctica de combinar e integrar actores, elementos y valores de múltiples áreas del saber, el conocimiento y la técnica práctica. A identificar sinergias, analogías, paradojas y enfoques desde múltiples puntos de vista y enfocados en distintos aspectos de los fenómenos y procesos que trabajamos.

Esta interdisciplinariedad, requiere de estímulo, estructura y exploración de los campos envueltos, con expertos específicos de cada uno aportando valor al objeto de estudio o trabajo. En el caso de la creatividad e innovación en la educación, la interdisciplinariedad debe ser estimulada por los docentes y facilitadores, para que los estudiantes puedan identificar dichas oportunidades y ser capaces de asociar los conocimientos y habilidades adquiridos en cada campo y combinarlos para un mejor desempeño. En esta línea, sería recomendable que los docentes coordinaran sus programas de clase, lecciones y actividades prácticas para propiciar oportunidades de interdisciplinariedad y aprendizaje combinado entre múltiples áreas del saber.

La interdisciplinariedad está fundamentada en marcos teóricos en el los cuales se indaga la existencia de coincidencias estructurales entre de las disciplinas. Y con respecto a la disponibilidad de las disciplinas científicas para la interdisciplinariedad se encuentra que desde su aspecto de comunicatividad existe una posible relación entre las didácticas disciplinares. En el trabajo interdisciplinario de la Enseñanza de los Contenidos Procedimentales en el Nivel Inicial (N I), y 1er Ciclo de la Educación General Básica de las disciplinas: Música, Matemáticas, Plástica, Ciencias Naturales. "Los nuevos procedimientos que se van aprendiendo se vinculan en la estructura cognoscitiva del alumno no sólo con otros procedimientos, sino también con el conjunto de componentes. Integrados y no aislados - que constituyen esa estructura (conceptos, con valores, con principios, etc.,) con lo cual un aprendizaje significativo de procedimientos redunda, a su vez, en una mejora de la capacidad global de aprender (Frega, 2007:70-126).

Categoría 4: Curriculum Transdisciplinar. La dimensión transdisciplinar del currículo plan- tean reflexiones que no son nuevas en el campo de la pedagogía, de la didáctica o incluso de la filosofía". En consecuencia, en la transversalidad y la educación integral la forma de entender la actividad pedagógica admite dos grandes enfoques, con aspiraciones diferentes, que se pueden encuadrar esquemáticamente bajo los temas enseñar y educar Por eso, para comprender el espíritu de los llamados ejes transversales es necesario retomar, por un lado, los planteamientos acerca de la educación en valores, y, por otro, el pensamiento sobre globalidad del conocimiento, junto a la relación de este con las conductas. Como bien se precisó en la propuesta arriba referenciadas, encontramos unas características que relacionan las autoras sobre el tema de la transversalidad, en los cuales destacan en primera instancia: valores, normas y actitudes que se plantean en los distintos ámbitos de la transversalidad y que tienen una relación profunda, ya que todos ellos apelan a principios universales y fundamentales como son la equidad (en contradicción con cualquier tipo de discriminación y dominación), la Solidaridad (frente a las distintas formas de explotación y de egoísmo), la Justicia (contra a situaciones de desprecio a los derechos individuales y colectivos), la Libertad (como situación que supere cualquier modo de esclavitud y de falta de reconocimiento del ámbito propio de decisión), la Salud (enfrenta la desvalorización del propio cuerpo y del bienestar general),etc.

Categoría 5: Curriculum Multidisciplinar. La multidisciplinariedad es un elemento clave para la creatividad y la innovación, así como un requisito para la interdisciplinariedad y la Transdisciplinariedad. Si bien puede sonar a un rebuscamiento innecesario, prefiero usar los tres términos para definir tres procesos o fenómenos distintos pero relacionados sobre el aprendizaje y la práctica holística del saber y las habilidades se puede decir que la multidisciplinariedad está orientada a la búsqueda de conocimiento, interés o desarrollo de habilidades en múltiples campos. La multidisciplinariedad es algo natural, común y que ocurre con cierta frecuencia. Por ejemplo, es común para los estudiantes en la practicar de deportes, la práctica musical, o cualquier otro lenguaje artístico, tomar clases de 
matemática y ciencias naturales en la educación primaria, o clases de física, química y literatura en la educación secundaria, y de ética, filosofía, matemáticas y lengua en los ciclos propedéuticos o generales de la educación superior. Pero la multidisciplinariedad no servirá de mucha ventaja, a menos que se logre conectar los saberes y valores de dichos campos.

En síntesis, la Multidisciplinariedad surge como resultado de dos motivaciones fundamentales: una académica (epistemológica) y la otra instrumental. La primera tiene como objetivo la reunificación del saber y el logro de un cuadro conceptual global; mientras que la segunda pretende investigar multilateralmente la realidad, por el propio carácter variado, multifacético y complejo de ésta y la necesidad de obtener un saber rápidamente aplicable, en consonancia con la creciente interrelación ciencia, tecnología y sociedad.

\section{CONCLUSIONES}

La propuesta del curriculum transversal, del aprendizaje para la vida, se dimensiona la acción social, como tema central de la orientación de los problemas y conflictos escolares, lo cual es de gran trascendencia para una educación integradora de lo humano, lo académico, lo tecnológico, etc. El hecho más generalizado es que estos temas la mayoría de las veces se trabajan de forma aislada y circunstancial, pues en la mayoría de los centros educativos aún no se hacen planteamientos de educación global, ni se hace un tratamiento didáctico continuado o suficientemente integrado en la actividad escolar. El carácter transversal de estas cuestiones implica que los mismos deben impregnar toda la práctica educativa y estar presente en las diferentes áreas curriculares.

En este contexto, se llega a la comprensión que los currículos "ínter" y "trans" disciplinario, son espacios donde interactúan críticamente las disciplinas antropológicas, educativas y sociales. Esta postura ayuda a adentrarnos en un contexto de incertidumbres y complejidades, que requiere no rigidizar la forma de ver $\mathrm{y}$ atender las disciplinas, y los fenómenos culturales en los contextos macro y micro, fundamentados teóricamente por los supuestos epistémicos y ontoló- gicos sociales de estudios performativos en el marco histórico cultural en el cual se desarrolla la génesis del arte, la creatividad y la cultura del contexto en el tiempo, y su implicación en la conciencia social de los contextos naturales, solo así se puede otro acabar la permanente cultura del colonialismo en el diseño y planeación del curriculum, y a su vez se atienda los valores e interés que tienen las comunidades por que se logren transformar sus prácticas y saberes, lo cual no se da si no se opta por acabar con la colonialidad del saber, del ser y del hacer (Lander Edgardo, 2000).

El curriculum transversal, visiona a la educación como un servicio público - bien social que tiene una función social; en ella se busca el acceso al conocimiento a la ciencia, a las nuevas tecnologías, a los bienes y valores de la cultura de manera integral. En este mismo orden contextual, se concibe en la sociedad el desarrollo de la formación integral, emocional, el conocimiento, valoración y respeto por los bienes valores personales y culturales. De igual forma, se apela a la cultura como artículo constitutivo del currículo, siendo éste un proceso global que se vive, se incorpora y se dinamiza día a día, en cada espacio abierto y en cada institución, ese proceso global que forma al individuo conscientemente como una persona comprometida con su proceso de desarrollo individual, comunitario, local y nacional.

La definición de la Integración de Saberes e Interdisciplinariedad, la Interdisciplinariedad es la interacción entre dos formas disciplinas que puede ir desde la simple comunicación de ideas hasta la integración reciproca de los conceptos fundamentales y de la teoría del conocimiento, de la metodología y de la enseñanza con finalidades de investigación o solución de problemas. Por su parte, la integración significa la unidad de las partes, tal que las partes quedan transformadas de alguna manera. Siguiendo al profesor López- Barajas (1997:111) la integración de saberes y la interdisciplinariedad son como dos caras de una moneda en la investigación educativa.

Por otro lado, la heurística hace referencia a la investigación misma, siendo en este caso la integración de saberes. El otro lado de la moneda 
seria la parte didáctica, es decir, el cómo hacer, esto sería la interdisciplinariedad. Por su parte, la Transdisciplinariedad sería la unificación de distintas ciencias con un solo método y un solo paradigma.

\section{REFERENCIAS}

Bernstein, B. (1985): la clasificación y enmarcación del conocimiento educativo, en Revista Colombiana de educación. 1er Semestre 1985.

Celma, Margarita, Ferrer Ramos, Ceip Vicente (2010). “Cantem Junts". Un Proyecto Interdisciplinario de recuperación del Folclor Musical del Matarranya. Val de Robles (Teruel).

Cox, G. (1998): “Teaching music in the UK secondary schools: Some life History perspectives". Comunicación presentada ante la American Education Research Association. San Diego.

Cremin, L. (1970): America education: The colonial experience 1607 - 1783. New York, Harper.

Fernández Batanero, José María (1992). La Organización de los contenidos en el marco de la atención de la diversidad. Universidad de Sevilla, España. (Consultado en Web, noviembre 6 de 2014).

Frega, Ana Lucia (2003). Arte, Música, Educación e Interdisciplinariedad. Resultados de una investigación en curso. Academia Nacional de Educación. Universidad Caece, Buenos Aires. República de Argentina.

(2007): Interdisciplinariedad Enfoques Didácticos para Educación General. Editorial Bonum, Buenos Aires, Argentina.

Freire, Paulo (1974). Educación y Cambio. Ed. Búsqueda. Buenos Aires.

García Canclini, Néstor (1984). Las Culturas Populares en el Capitalismo. Ed. Nueva Imagen. México.

--------- (1990). Culturas Híbridas. Editorial Grijalbo. México.

(1995). Consumidores y Ciudadanos. Conflictos multiculturales de la globalización. Editorial Grijalbo. México.
Habermas, Jürgen (1948). El discurso filosófico de la modernidad. Madrid, Taurus.

Flórez, Rafael (1994): Hacía una pedagogía del conocimiento. Bogotá Mc Graw Hill,

--------- (1999): Hacia la pedagogía del convencimiento. Santa Fe de Bogotá. Mcgraw Hill, ----(1996) Citado por Facultad de Ciencias de la Educación: Pedagogía en los programas de pregrado presenciales y semipresenciales. Universidad del Atlántico. Barranquilla.

Gimeno Sacristán, J (1988). El curriculum: una reflexión sobre la práctica. Madrid. Morata.

Grossman Pam, Susan Stodolsky (1997). Content as Context: The Role of School Subjects in Secondary School Teaching. Journal Articles. Stanford, CA 94305-3084

Stanford University. (Consulta en la Web, 20 de septiembre, 2014).

Imbernón, F. (1999): “La formación y la profesionalización en la función pedagógica”. En V.S. Universidad de Granada.

Lander, Edgardo. (2000). La Colonialidad del Saber: Eurocentrismo y Ciencias Sociales. Perspectivas latinoamericanas. CLACSO. Consejo Latinoamericano de Ciencias Sociales. Buenos Aires.

López - Barajas Zayas, Emilio (1997): Integración de Saberes e Interdisciplinariedad. Actas y Congresos. Universidad Nacional de Educación a Distancia, Madrid, España.

López Jiménez, Nelson Ernesto (2002); Retos Para la Construcción Curricular. De la Certeza al Paradigma de la Incertidumbre Creativa. Editorial: Cooperativa Editorial Magisterio, Bogotá, Colombia.

La Ley Orgánica de Mejora de la Calidad Educativa (LOMCE). Séptima reforma en profundidad del sistema educativo de la democracia. EFE Madrid 21/09/2012.

LOGSE (LEY ORGÁNICA 9/1992, de 23 de diciembre). Transferencia de competencias a Comunidades Autónomas que accedieron a la autonomía por la vía del artículo 143 de la Constitución, Madrid, España. 
Magendzo, Abraham. (1986). Currículum y cultura en América Latina; Publicada por el Programa Interdisciplinario de investigación en educación. 1era edición. Santiago de Chile.

-------. (1995). Currículum, Educación para la Democracia. Instituto para el Desarrollo de la Democracia Luis Carlos Galán. Santafé de Bogotá, D.C., Colombia.

(2003). Transversalidad y Currículum. Bogotá: cooperativa Editorial magisterio. 1era edición.

Michael, Mark (2002). Connecting Comics to Curriculum Strategies for Grades 6 - 12. For Karen W. Gavigan and Mindy Tomasevich. Library of Congress. Publication Data.

Morín, E. (1994). Introducción al Pensamiento Complejo. Gedisa. Barcelona.

Novoa, A. (1999): "La nueva cuestión central de los profesores. Excesos de discursos, pobreza de prácticas". Cuadernos de pedagogía, 286, 102 108.

Ocaña Almudena (2006). Desarrollo Profesional de las Maestras de Educación Musical desde una Perspectiva Biográfico - Narrativa. Revista Electrónica Complutense de Investigación en Educación Musical. Volumne 3. Número. Universidad de Granada, España.

Ortega y Gasset (1937; edición electrónica 2004). La rebelión de las masas. Disponible: www.laeditorialvirtual.com.ar/.../Ortega_y_Gasset/Ortega_LaRebelionDeLasMasas0

1.htm.

Ortiz Castro, Hellver Jazzyd. (1999). Notas de la UNESCO sobre políticas de la primera infancia. Interdisciplinariedad: problemas de la enseñanza y de la investigación en las Universidades.

Ortiz Castro, Hellver Jazzyd (2004). Revista de psicodidactica, número 017. Universidad del País Vasco. Victoria - Gasteiz, España.

Perera Cumerma, Fernando (1986). La Formación Interdisciplinaria de los Profesores: Una Necesidad del Proceso De Enseñanza-Aprendizaje de las Ciencias. ISP "Enrique José Varona".
La Habana (1992).

(1986). Las relaciones interdisciplinarias de la Física en las carreras de Química y de Biología de la Licenciatura en Educación. Ponencia a la Reunión Científica de Profesores de la Facultad de C. Naturales. La Habana.

Pérez Herrera, Manuel Antonio (2008): Una Pedagogía de Salvaguardia del Carnaval de Barranquilla. (Capítulo de Libro Pensar el Caribe II). Editorial Universidad del Atlántico.

(2011): El Carnaval de Barranquilla como fuente de Pedagogía. Revista El Artista. Número 4, noviembre. Universidad de Pamplona.

(2012). Integración de la música a la estructura curricular de los programas académicos de educación artística musical de la Universidad del Atlántico. Tesis Doctoral. Doctorado en Ciencias de la Educación. Rudecolombia- Cade Universidad de Caldas, Manizales, Colombia.

----------- (2013).Integración de Una Pedagogía Educación Social al Contexto Universitario. Revista Escenarios Volumen $11 \mathrm{~N}^{\circ} 2$. Universidad Autónoma del Caribe, Barranquilla.

(2014). La Música como Eje Transversal del Desarrollo del Conocimiento. Texto inédito. Producto de Tesis Doctoral: Integración de la música a la estructura curricular de los programas académicos de educación artística musical de la Universidad del Atlántico. Tesis Doctoral. Doctorado en Ciencias de la Educación. Rudecolombia- Cade Universidad de Caldas, Manizales, Colombia.

Proyecto Académico Curricular (1999): Elementos Propedéuticos. Revista Facultad de Ciencias de la Educación, Universidad del Atlántico, Barranquilla, Colombia.

Revista Eufonía. Didáctica de la Música. Editorial Grao/. Núm. 49. Barcelona (España).

Revista Mente y Cerebro (nov.13: Número 2005). Fondo de Cultura Económica, México.

Román Pérez, Mariniano y Diez López, Eloísa (1994): Curriculum y Enseñanza Una Didáctica 
Centrada en Procesos. Universidad Complutense de Madrid. Editorial EOS, Madrid (España).

Sarró Maluquer, Ramón (1988): Ciudad saludable: su función y su futuro. Revista Anthropos. Traducido al Inglés por Sarró Maluquer, Ramón. Editorial EOS, Madrid (España).

Stenhouse, L (1984): Investigación y Desarrollo del Curriculum. Edición Morata. Madrid, España.

Taba, Hilda (1974). Elaboración del Currículo: Teoría y Práctica, proporcionara estímulos intelectuales y profesionales a los docentes de college* y a quienes dirigen la tarea educacional. Editorial Troquel S.A. Buenos Aires.

Times Suplement Educacional (1998). Revista Electrónica, del 24 de abril de 1998. Londres.

Torres, J. (1994). Globalización e Interdisciplinariedad: el currículum integrado. Ed. Morata.
Madrid, España.

Vega Rebeca, Michel / Corral Ruso, Roberto (2006). Revista: Curriculum Dezembrol, Año / Vol. 2, numero 003. Pontificia Universidad católica de Sao Paulo, Brasil.

Yus Ramos, Rafael (2001): Educación Integral Una Educación Holística para el Siglo XXI. Editorial Desclée de Brouwer, S.A. Bilbao (España).

------------ (1996). Hacia una Educación Global desde la Transversalidad. Edición: Grupo Anaya, S.A. Funlabrada. Madrid (España).

William "Bill" Bobbit, (1918). Main St., Ringtown, passed away peacefully Monday at Shenandoah Manor Nursing Center where he was recently a guest.

(En Born in Mahanoy City, Aug. 30, 1918, he was a son of the late William and Anastasia Sakaloski Bobbit). 